\title{
Effect of Androgen-deprivation Therapy on Bone Mineral Density in Japanese Patients with Prostate Cancer
}

\author{
YOSHIYUKI MIYAZAWA, YOSHITAKA SEKINE, TAKAHIRO SYUTO, MASASHI NOMURA, \\ HIDEKAZU KOIKE, HIROSHI MATSUI, YASUHIRO SHIBATA, KAZUTO ITO and KAZUHIRO SUZUKI \\ Department of Urology, Gunma University Graduate School of Medicine, Maebashi, Japan
}

\begin{abstract}
Background/Aim: To evaluate bone mineral density (BMD) in Japanese patients with prostate cancer (PCa) after administering androgen deprivation therapy (ADT) for 2 years. Patients and Methods: A total of 84 Japanese patients with PCa were enrolled in this study during the period 2008-2011. BMD was measured by dual energy X-ray absorptiometry, every 6 months. The fracture risk assessment tool (FRAX) score was calculated before starting ADT. We evaluated the change in BMD over a 2year period and the relationship between this change, the FRAX score, and the estimated glomerular filtration rate (eGFR). Results: Compared to baseline, BMD decreased by $2.50 \%$ at 6 months after ADT, by $4.28 \%$ after 12 months, by $5.34 \%$ after 18 months, and by $6.16 \%$ after 2 years (all $p<0.05)$. Multivariate analysis revealed that the eGFR, according to a threshold rate of $73.5 \mathrm{ml} / \mathrm{min}$, was a significant factor in BMD. Conclusion: Lumbar BMD in Japanese patients with PCa decreased by $4.28 \%$ at 1 year after ADT and by $6.16 \%$ after 2 years. We found a correlation between the decrease in BMD and the eGFR before initiating $A D T$, suggesting a small BMD reduction in patients with PCa who have good renal function.
\end{abstract}

Prostate cancer (PCa) is one of the most common carcinomas in developed countries, including Japan (1). $\mathrm{PCa}$ is known to be an androgen-dependent cancer and controlling androgen levels is thus effective for its suppression. Surgery and radiation therapy are often

This article is freely accessible online.

Correspondence to: Yoshiyuki Miyazawa, Department of Urology, Gunma University Graduate School of Medicine, 3-9-22 Showamachi, Maebashi, Gunma 371-8511, Japan. Tel: +81 272208306, Fax: +81272208307, e-mail: miya.yoshi@hotmail.co.jp

Key Words: Prostate cancer, androgen-deprivation therapy, bone mineral density, chronic kidney disease. applied for curative treatment of topical PCa, but androgen deprivation therapy (ADT) is usually used in Japan as the initial therapy in combination with radiation, and in cases that are refractory to surgery. While ADT is highly effective, various complications associated with its longterm use may be problematic, including osteoporosis accompanying a decrease in bone mineral density (BMD) (2-4). A decrease in BMD is associated with an increased incidence of bone fracture, and various complications are believed to increase the mortality rate. A reduction in muscle mass due to ADT may also contribute to an increased risk of falls (5-6). Previous studies done in the United States showed that BMD decreased by $2-8 \%$ in the first year after ADT initiation $(3,7,8)$. The fracture risk assessment tool (FRAX) score has been used to predict the incidence of bone-related events. The FRAX score was developed by the World Health Organization to predict fracture risk in patients according to the presence of clinical risk factors, alone or in combination with femoral neck BMD (9). It is a computer-based algorithm that calculates the 10-year probability of hip and major osteoporotic fractures (e.g., clinical spine, forearm, hip, or shoulder fracture) according to age, sex, body mass index, and clinical risk factors $(10,11)$. No study has reported on the relationship between the FRAX score before ADT and the change in BMD after initiating ADT treatment in Japanese patients with PCa. In this study, we measured the effect of administering a luteinizing hormone-releasing hormone (LHRH) agonist on BMD in patients with PCa over 2 years and examined the relationship between the change in BMD and FRAX score before treatment. Chronic kidney disease (CKD) can lead to disturbed mineral homeostasis, increasing or decreasing the circulating levels of calcium, phosphorus, fibroblast growth factor 23 , vitamin D, and parathyroid hormone. This effect on mineral homeostasis may be associated with increased bone fragility $(12,13)$. Therefore, we examined renal function before the start of ADT and examined the degree of change in BMD after administration of ADT. 


\section{Patients and Methods}

We conducted an investigator-initiated, prospective, single-arm, open-label trial. The primary objective of the study was to evaluate the change in lumbar spine BMD after administration of ADT for 2 years in Japanese patients. A total of 103 Japanese patients with PCa were enrolled in this study during the period 2008-2011. All patients were pathologically diagnosed with PCa and followed-up at Gunma University Hospital (Gunma, Japan). A retrospective study was conducted on 84 of the patients, who were followed for 2 years. Of the 19 patients who were lost to follow up, 1 died of rectal cancer and the other 18 were transferred to another institution. The characteristics of the 84 patients are shown in Table I. The mean age of the 84 patients was 70.3 years; the median prostate-specific antigen (PSA) level before administration of ADT was $10.1 \mathrm{ng} / \mathrm{ml}$. The clinical stages of the patients were T1cNOM0 $(n=30)$, T2NOM0 $(n=23)$, T3N0M0 $(n=29)$, and T4N1M1 $(n=2)$. Two patients had bone metastases and lymph node metastasis. Eighteen patients underwent combined-androgen blockade $(\mathrm{CAB})$ with bicalutamide. Among the 84 cases studied, no patient was administered medications for osteoporosis (bisphosphonates, vitamin D, calcium preparations, etc.) during the 2-year study period. All patients were administered leuprolide as ADT. A dose of $3.75 \mathrm{mg}$ was administered only at the start of treatment, with $11.25 \mathrm{mg}$ being administered every 3 months thereafter. Seventy patients $(83.3 \%)$ underwent radiation treatment (external beam radiation therapy) for curative purposes; the other fourteen patients received ADT only. No events, such as fractures, occurred in any case during the observation period. In total, 5 patients $(6.0 \%)$ had diabetes, 13 (15.5\%) had heart disease, $17(20.2 \%)$ had hypertension, and 4 $(4.8 \%)$ had a history of other carcinoma treatments. The estimated glomerular filtration rate (eGFR), used as an indicator of kidney function, was calculated by substituting sex, age, weight, and serum creatinine level $(\mathrm{mg} / \mathrm{ml})$ data into the Cockcroft-Gault formula. The Ethics Committee for Clinical Studies of Gunma University Graduate School of Medicine approved this study, and written consent was obtained from all enrolled patients.

BMD measurements. BMD was measured in our hospital during the period 2008-2011 by dual energy X-ray absorptiometry (DXA) using a Hologic DXA scanner (Hologic, Bedford, MA, USA). The $\mathrm{BMD}$, in grams per square centimeter, was measured in the lumbar spine. We measured BMD by DXA at pre-treatment and at 6,12 , 18 , and 24 months after starting ADT treatment. The BMD after initiating treatment was compared with the pre-treatment BMD.

FRAX scoring. The FRAX score was calculated before starting ADT. The FRAX tool (https://www.sheffield.ac.uk/FRAX/tool.jsp? lang=jp) was used to compute the probability of a major osteoporotic event or a hip fracture: in this study, we used the Japanese version of the instrument. Date of birth, weight $(\mathrm{kg})$, height $(\mathrm{cm})$, and various clinical risk factors (history of fracture, history of hip fracture in a parent, tobacco smoker, current use of glucocorticoids, rheumatoid arthritis, secondary osteoporosis, and daily consumption of $\geq 3$ units of alcohol; all yes/no responses) were entered into the FRAX tool, and the fracture probability was calculated for each subject. Because we could not obtain the femoral neck BMD data, we did not enter this variable into FRAX. ADT was performed, and the questionnaire items pertaining to the presence or absence of secondary osteoporosis examined, in all cases.
Table I. Clinical characteristics of the patients.

\begin{tabular}{|c|c|c|}
\hline \multicolumn{3}{|l|}{ Characteristic } \\
\hline \multicolumn{3}{|l|}{ No. of patients } \\
\hline \multicolumn{2}{|c|}{ Age (years, mean \pm SD) } & $70.3 \pm 5.4$ \\
\hline \multicolumn{2}{|c|}{ Initial PSA level $(\mathrm{ng} / \mathrm{ml}$, median \pm SD $)$} & $10.1 \pm 53.3$ \\
\hline \multicolumn{2}{|c|}{ Height $(\mathrm{cm}$, mean \pm SD) } & $163.8 \pm 5.5$ \\
\hline \multicolumn{2}{|c|}{ Body weight $(\mathrm{kg}$, mean $\pm \mathrm{SD})$} & $62.3 \pm 8.3$ \\
\hline \multicolumn{2}{|c|}{$\mathrm{BMI}(\operatorname{mean} \pm \mathrm{SD})$} & $23.0 \pm 2.8$ \\
\hline & No. of patients & Proportion of patients \\
\hline \multicolumn{3}{|l|}{ Stage } \\
\hline T1cN0M0 & 30 & $35.7 \%$ \\
\hline T2N0M0 & 23 & $27.4 \%$ \\
\hline T3N0M0 & 29 & $34.5 \%$ \\
\hline T4N1M1 & 2 & $2.4 \%$ \\
\hline \multicolumn{3}{|l|}{ GS } \\
\hline 6 & 3 & $3.6 \%$ \\
\hline 7 & 36 & $42.9 \%$ \\
\hline 8 & 21 & $25.0 \%$ \\
\hline 9 & 20 & $23.8 \%$ \\
\hline 10 & 4 & $4.8 \%$ \\
\hline Surgery plus radiation & 70 & $83.3 \%$ \\
\hline ADT alone & 14 & $16.7 \%$ \\
\hline \multicolumn{3}{|l|}{ Medical history } \\
\hline Diabetes & 5 & $6.0 \%$ \\
\hline Heart disease & 13 & $15.5 \%$ \\
\hline Hypertension & 17 & $20.2 \%$ \\
\hline Other carcinoma & 4 & $4.8 \%$ \\
\hline
\end{tabular}

PSA: Prostate-specific antigen; SD: standard deviation; BMI: body mass index; GS: Gleason score; ADT: androgen deprivation therapy.

Statistical analysis. Numerical data were compared using Student's $t$-test. Correlations among variables were determined using binomial logistic regression analysis. In all analyses, $p$-values $<0.05$ were considered significant. We conducted the statistical analysis using SPSS software (ver. 24.0; IBM Corp., Armonk, NY, USA).

Ethics approval and consent to participate. The Ethics Committee for Clinical Studies of the Gunma University Hospital approved this study, and written consent was obtained from all of the enrolled patients. It was performed in accordance with the principles of the Declaration of Helsinki. All patients provided written informed consent.

\section{Results}

The pre-treatment parameters of the 84 cases are summarized in Table II. Mean lumbar spine BMD was $1.025 \pm 0.200$ $\mathrm{mg} / \mathrm{cm} 2$. Mean serum creatinine was $0.80 \pm 0.18 \mathrm{mg} / \mathrm{ml}$, and the mean eGFR calculated based on serum creatinine was $73.5 \pm 5.40 \mathrm{ml} / \mathrm{min}$. Among all patients, the median 10-year probability of fracture according to the risk factors entered into FRAX was $23.0 \pm 2.8 \%$. The median 10 -year probability of a major osteoporotic fracture was $9.6 \pm 3.4 \%$. The median 
Table II. Baseline characteristics of the patients.

\begin{tabular}{lc}
\hline Characteristic & \\
\hline BMD $\left(\mathrm{mg} / \mathrm{cm}^{2}\right.$, mean $\left.\pm \mathrm{SD}\right)$ & $1.025 \pm 0.20$ \\
Serum creatinine $(\mathrm{mg} / \mathrm{ml}$, median $\pm \mathrm{SD})$ & $0.80 \pm 0.18$ \\
eGFR (ml/min, median $\pm \mathrm{SD})$ & $73.5 \pm 5.40$ \\
\hline FRAX item & No. of patients $(\%)$ \\
\hline Previous fracture & $4(4.8 \%)$ \\
Parent fractured hip & $3(3.6 \%)$ \\
Current smoker & $20(23.8 \%)$ \\
Glucocorticoid & $1(1.2 \%)$ \\
Rheumatoid arthritis & $0(0 \%)$ \\
Alcohol use ( $\geq 3$ units/day) & $29(34.5 \%)$ \\
\hline FRAX results & $\%$, median $\pm \mathrm{SD}$ \\
\hline 10-year probability of fracture & $23.0 \pm 2.8$ \\
10-year probability of a major osteoporotic fracture & $9.6 \pm 3.4$ \\
10-year probability of hip fracture & $3.6 \pm 2.6$ \\
\hline
\end{tabular}

BMD: Bone mineral density; eGFR: estimated glomerular filtration rate; FRAX: fracture risk assessment tool.

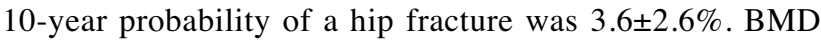
tended to decrease with time, but the decline at 1 year after treatment tended to be proportionally larger than that between 1 and 2 years after treatment.

Table III shows changes in BMD during the 2-year period after starting ADT. Relative to baseline, BMD decreased by $2.50 \%$ at 6 months after ADT, by $4.28 \%$ at 12 months, by $5.34 \%$ at 18 months, and by $6.16 \%$ at 2 years (all $p<0.05$ ).

Univariate and multivariate analyses were performed to determine whether or not the decrease in BMD at 2 years after ADT was less than $6.16 \%$ : the results are shown in Table IV. The only factor that remained significant in the multivariate analysis was the eGFR $(\geq 73.5 \mathrm{vs} .<73.5 \mathrm{ml} / \mathrm{min})$ No significant correlation was detected between the rate of bone-related events (calculated by reference to the FRAX score) and the rate of change in BMD.

\section{Discussion}

ADT causes a reduction in BMD, thus resulting in an increased frequency of osteoporosis and fractures. Previous reports showed that BMD decreases by $2-8 \%$ in the first year after ADT initiation $(3,4,7)$. The BMD decline in men treated with ADT may be greater compared with the $2.5 \%$ decrease per year reported for post-menopausal women (14). Maillefert et al. showed that lumbar spine BMD decreased by $7.1 \%$ after ADT treatment combined with 18 months of LHRH agonist administration in 12 patients with PCa (7). Miyaji et al. showed that, in 27 Japanese patients without
Table III. Absolute change $\left(\mathrm{mg} / \mathrm{cm}^{2}\right)$ and percent change in bone mineral density from baseline.

\begin{tabular}{lccccc}
\hline & Pre & $6 \mathrm{~m}$ & $12 \mathrm{~m}$ & $18 \mathrm{~m}$ & $24 \mathrm{~m}$ \\
\hline Mean & 1.025 & 0.996 & 0.961 & 0.961 & 0.958 \\
SD & 0.200 & 0.196 & 0.194 & 0.184 & 0.191 \\
Minimum & 0.595 & 0.511 & 0.511 & 0.515 & 0.498 \\
Maximum & 1.572 & 1.574 & 1.427 & 1.427 & 1.541 \\
Percent change from baseline & - & -2.50 & -4.28 & -5.34 & -6.16 \\
\hline
\end{tabular}

SD: Standard deviation.

Table IV. Factors predicting a decrease in BMD, at 2 years after initiation of ADT, of less than 6.16\%: results of univariate and multivariate analyses.

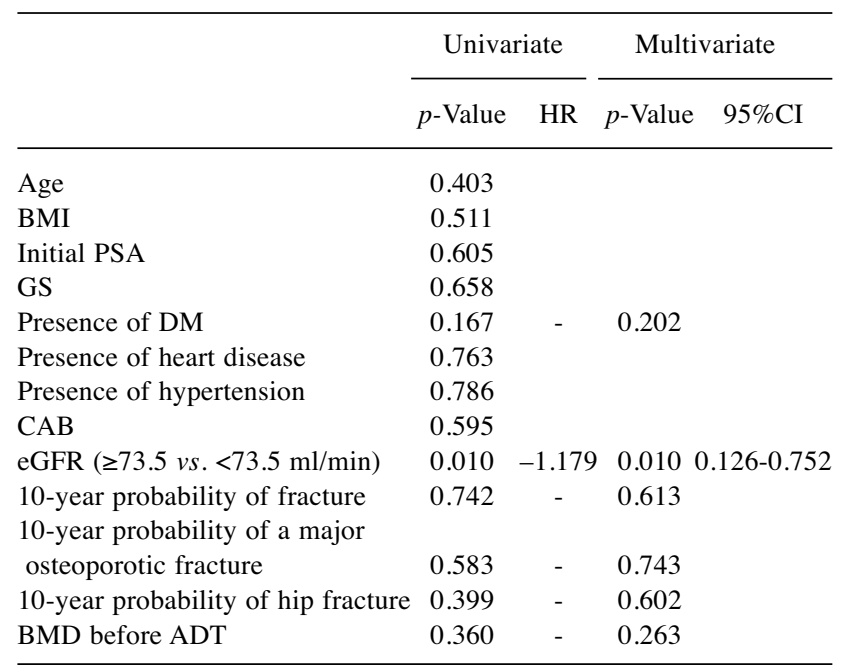

BMI: Body mass index; PSA: prostate-specific antigen; GS: Gleason score; DM: diabetes mellitus; $\mathrm{CAB}$ : combined androgen blockade; eGFR: estimated glomerular filtration rate; BMD: bone mineral density; ADT: androgen deprivation therapy; HR: hazard ratio; $\mathrm{CI}$ : confidence interval.

bone metastasis in 2004, the median lumbar spine BMD decreased significantly, from 0.966 to $0.937 \mathrm{~g} / \mathrm{cm}^{3}$ $(p=0.047)$, after 2 years of ADT (15). Based on these data, ADT for 2 years resulted in an approximately $3 \%$ reduction in BMD. Thus, there was an almost two-fold difference in the rate of reduction in BMD after ADT initiation between Miyaji et al. and the present study. Differences in sample size (84 cases in the present study, 27 cases in Miyaji et al.) and patient characteristics may have had an effect, and our results were similar to those of western studies (2-8).

With reference to the likelihood of fractures (as determined by FRAX), it may be useful to consider osteoporosis treatment in patients undergoing ADT treatment $(9,10)$. Prior 
to our analysis, we predicted that patients with high fracture risk, as calculated by FRAX, would show a greater decline in BMD, but the results did not support this. A logistic binomial analysis was conducted to examine whether there was a correlation between FRAX fracture risk and BMD; however, no significant correlation was found, suggesting that the risk of fracture calculated by FRAX is to calculate the possibility of the last fracture, indicating that it does not correlate with the decrease in BMD. As there were no bone-related events among our study population during the observation period, such as fractures, it was not possible to examine the correlations among fracture risk on FRAX, BMD, and bonerelated events. We aim to extend the observation period in future studies. Multivariate analysis revealed that the eGFR (median $=73.5 \mathrm{ml} / \mathrm{min}$ ) at ADT initiation was related to the degree of decrease in BMD. Regardless of whether or not ADT is administered, bone management of patients with CKD is important because kidney function is related to bone mineral content $(12,13)$. However, the optimal eGFR cutoff value for predicting reduced BMD is unknown. Therefore, a future study is necessary to ascertain this.

This study had certain limitations. For example, it is necessary to measure femoral neck BMD when calculating the FRAX score. However, our unique results regarding changes in BMD over 2 years following ADT initiation, in a comparatively large sample of Japanese patients with $\mathrm{PCa}$, are important for informing future bone management of such patients.

\section{Conclusion}

We found that the lumbar BMD of Japanese patients with PCa who underwent ADT decreased by $4.28 \%$ at 1 year and by $6.16 \%$ at 2 years. In addition, multivariate analysis showed that the decrease in BMD was correlated with the eGFR before initiating ADT, suggesting a small BMD reduction in patients with $\mathrm{PCa}$ and good renal function.

\section{Conflicts of Interest}

Yoshiyuki Miyazawa, Takahiro Syuto, Yoshitaka Sekine, Masashi Nomura, Hidekazu Koike, Hiroshi Matsui, Yasuhiro Shibata and Kazuto Ito declare that there are no conflicts of interest that could be perceived as prejudicing the impartiality of the research reported. Kazuhiro Suzuki is a recipient of research grants and honoraria from Takeda Pharmaceutical Co. Ltd. and Astellas Pharma Inc.

\section{Acknowledgements}

None.

\section{References}

1 Ito K: Prostate cancer in Asian man. Nat Rev Urol 11: 197-212, 2014.
2 Bergström I, Gustafsson H, Sjöberg K and Arver S: Changes in bone mineral density differ between gonadotrophin-releasing hormone analogue- and surgically castrated men with prostate cancer: a prospective, controlled, parallel-group study. Scand J Urol Nephrol 38: 148-152, 2004.

3 Berruti A, Dogliotti L, Terrone C, Cerutti S, Isaia G, Tarabuzzi R, Reimond G, Mari M, Ardissone P, De Luca S, Fasolis G, Fontana D, Rossetti SR and Angell A: Changes in bone mineral density, lean body mass and fat content as measured by dual energy x-ray absorptiometry in patients with prostate cancer without apparent bone metastases given androgen deprivation therapy. J Urol 167: 2361-2367, 2002.

4 Daniell HW, Dunn SR, Ferguson DW, Lomas G, Niazi Z and Stratte PT: Progressive osteoporosis during androgen deprivation therapy for prostate cancer. J Urol 163: 181-186, 2000.

5 Smith MR: Changes in fat and lean body mass during androgendeprivation therapy for prostate cancer. Urology 63: 742-745, 2004.

6 Smith MR, Finkelstein JS, McGovern FJ, Zietman AL, Fallon MA, Schoenfeld DA and Kantoff PW: Changes in body composition during androgen deprivation therapy for prostate cancer. J Clin Endocrinol Metab 87: 599-603, 2002.

7 Maillefert JF, Sibilia J, Michel F, Saussine C, Javier RM and Tavemier C: Bone mineral density in men treated with synthetic gonadotropin-releasing hormone agonists for prostatic carcinoma. J. Urol 161: 1219-1222, 1999.

8 Daniell HW, Dunn SR, Ferguson DW, Lomas G, Niazi Z and Stratte PT: Progressive osteoporosis during androgen deprivation therapy for prostate cancer. J Urol 163: 181-186, 2000.

9 Kanis JA, Oden A, Johansson H, Borgstrom F, Strom O and McCloskey E: FRAX and its applications to clinical practice. Bone 44(5): 734-743, 2009.

10 Johansson H, Kanis JA, Oden A, Johnell O and McCloskey E: BMD, clinical risk factors and their combination for hip fracture prevention. Osteoporosis Osteoporos Int 20(10): 1675-1682, 2009.

11 Kuruvilla K, Kenny AM, Raisz LG, Kerstetter JE, Feinn RS and Rajan TV: Importance of bone mineral density measurements in evaluating fragility bone fracture risk in Asian Indian men. Osteoporosis Int J 22(1): 217-221, 2011.

12 KDIGO: Clinical practice guideline for the evaluation and management of chronic kidney disease. Kidney Int Suppl 3: 1962, 2013.

13 KDIGO: Clinical practice guideline for the diagnosis, evaluation, prevention, and treatment of Chronic Kidney Disease-Mineral and Bone Disorder (CKD-MBD). Kidney Int Suppl 113(3): 1962, 2009.

14 Seifert-Klauss V, Fillenberg S, Schneider H, Luppa P, Mueller $\mathrm{D}$ and Kiechle M: Bone loss in premenopausal, perimenopausal and postmenopausal women: results of a prospective observational study over 9 years. Climacteric 15: 433-440, 2012.

15 Miyaji Y, Saika T, Yamamoto Y, Kusaka N, Arata R, Ebara S, Nasu Y, Tsushima T and Kumon H: Effects of gonadotropinreleasing hormone agonists on bone metabolism markers and bone mineral density in patients with prostate cancer. Urology 64: 128-131, 2004. 